\title{
A model for embedding high-fidelity simulation training within core medical training (soon to be internal medicine training)
}

\author{
Authors: Jamie Kitt, ${ }^{A}$ Ishita Patel ${ }^{B}$ and Alex Saunders ${ }^{C}$
}

\begin{abstract}
Aims
Feedback obtained from the General Medical Council survey of trainees within Oxford Deanery, now Health Education Thames Valley (HETV), from 2014 to 2016, supplemented by local surveys of Frimley Health's outgoing cohorts of core medical trainees (CMTs), highlighted a dire lack of procedural exposure across CMT programmes both locally and nationwide. We aimed to develop and embed a sustainable simulation training programme tailored to the 'essential and desirable procedures' and 'emergency presentations' outlined in the Royal College of Physicians / Joint Royal Colleges of Physicians Training Board curriculum for core medical trainees which provided them with the necessary skill set to develop as competent practitioners in this vital area of medical practice
\end{abstract}

\section{Methods}

All CMTs at Wexham Park Hospital between 2016-2018 have taken part in two simulation days, the first focused on the 'essential skills' laid out in the curriculum and the second on the emergency presentations. This has rolled on a 6-monthly basis to capture anyone missing the first run / newcomers to the trust, and in autumn 2017 was expanded to CMTs across HETV. Serial pilot programmes were run between 2014-2016 and patient feedback was obtained to develop the simulation programme initiated in 2016. Formal written feedback was obtained from each of the participants prior to, and immediately after, completion of each day of the simulation programme to gauge competence and confidence pre- and post- training, and to allow further refinement of the programme. Completion of the simulation training resulted in access to 'real-life' training lists, in which trainees, under the supervision and guidance of nominated and accredited trainers were given the opportunity to translate the simulation skills into

Authors: ${ }^{A}$ Cardiology and general internal medicine ST6 and chief registrar from 2016-2017, Oxford University Hospitals NHS Foundation Trust, Oxford; ${ }^{B}$ Royal College of Physicians tutor, Frimley Health NHS Foundation Trust, Slough, UK; ${ }^{C}$ lead for TEL and simulation services 'real-life' level 2 competence by the end of the 2 years of core medical training.

\section{Results}

We designed tailored and fully anonymised feedback forms for each day of the simulation programmes. Summated Likert scores from pre- and post-course demonstrated an average of 1-2 degrees of improvement on the Likert scales. The qualitative feedback was also extremely positive for both days and additional verbal feedback indicated a strong desire for this programme to continue and expand.

\section{Conclusion}

Following the success of this project at a local level in Frimley Health NHS Foundation trust we are expanding the programme deanery-wide, with assistance from the simulation lead for HETV. As of August 2018 it will be offered to all CMT trainees across HETV based at QuEST in Frimley and OxSTaR in Oxford via a centralised online booking portal, which is currently in development. Further refinements are being made in line with feedback from the first 2-years pilot including new simulation scenarios co-developed with KSS Deanery. A 'Reg-ready course' for core medical trainee year 2-specialty trainee year 3 trainees will also be added in due course, which has just been successfully piloted in May and Autumn 2017 at QUEST. This simulation programme provides a model for developing and embedding a successful and vital training programme, and then how to centralise resources and faculty so that all trainees across a deanery can be provided with this 'essential' training free of charge as of August 2018.

\section{Conflict of interest statement}

No conflicts of interest to declare. 